\title{
NON-UNIQUENESS OF CONVEX BODIES WITH PRESCRIBED VOLUMES OF SECTIONS AND PROJECTIONS
}

\author{
FEDOR NAZAROV, DMITRY RYABOGIN AND ARTEM ZVAVITCH
}

\begin{abstract}
We show that if $d \geq 4$ is even, then one can find two essentially different convex bodies such that the volumes of their maximal sections, central sections, and projections coincide for all directions.
\end{abstract}

\section{INTRODUCTION}

As usual, a convex body $K \subset \mathbb{R}^{d}$ is a compact convex subset of $\mathbb{R}^{d}$ with non-empty interior. We assume that $0 \in K$. We consider the central section function $A_{K}$ :

$$
A_{K}(u)=\operatorname{vol}_{d-1}\left(K \cap u^{\perp}\right), \quad u \in \mathbb{S}^{d-1},
$$

the maximal section function $M_{K}$ :

$$
M_{K}(u)=\max _{t \in \mathbb{R}} \operatorname{vol}_{d-1}\left(K \cap\left(u^{\perp}+t u\right)\right), \quad u \in \mathbb{S}^{d-1},
$$

and the projection function $P_{K}$ :

$$
P_{K}(u)=\operatorname{vol}_{d-1}\left(K \mid u^{\perp}\right), \quad u \in \mathbb{S}^{d-1} .
$$

Here $u^{\perp}$ stands for the hyperplane passing through the origin and orthogonal to the unit vector $u, K \cap\left(u^{\perp}+t u\right)$ is the section of $K$ by the affine hyperplane $u^{\perp}+t u$, and $K \mid u^{\perp}$ is the projection of $K$ to $u^{\perp}$. Observe that $A_{K} \leq M_{K} \leq P_{K}$. It is well known, Ga], that for origin-symmetric bodies each of the functions $M_{K}=A_{K}$ and $P_{K}$ determines the convex body $K \subset \mathbb{R}^{d}$ uniquely. More precisely, either of the conditions

$$
M_{K_{1}}(u)=M_{K_{2}}(u) \quad \forall u \in \mathbb{S}^{d-1},
$$

and

$$
P_{K_{1}}(u)=P_{K_{2}}(u) \quad \forall u \in \mathbb{S}^{d-1},
$$

implies $K_{1}=K_{2}$, provided $K_{1}, K_{2}$ are origin-symmetric and convex.

In this paper, we address the (im)possibility of analogous results for not necessarily symmetric convex bodies.

It is well known, $\overline{\mathrm{BF}}$, that on the plane there are convex bodies $K$ that are not Euclidean discs, but nevertheless satisfy $M_{K}(u)=P_{K}(u)=1$ for all $u \in \mathbb{S}^{1}$. These are the bodies of constant width 1 .

Key words and phrases. Convex body, sections, projections.

The first author is supported in part by U.S. National Science Foundation Grant DMS-0800243. The second and third named authors are supported in part by U.S. National Science Foundation Grant DMS-1101636. 
In 1929 T. Bonnesen asked whether every convex body $K \subset \mathbb{R}^{3}$ is uniquely defined by $P_{K}$ and $M_{K}$, (see $[\mathrm{BF}$, page 51 ). We note that in any dimension $d \geq 3$, it is not even known whether the conditions $M_{K} \equiv c_{1}, P_{K} \equiv c_{2}$ are incompatible for $c_{1}<c_{2}$.

In $1969 \mathrm{~V}$. Klee asked whether the condition $M_{K_{1}} \equiv M_{K_{2}}$ implies $K_{1}=K_{2}$, or, at least, whether the condition $M_{K} \equiv c$ implies that $K$ is a Euclidean ball, see [Kl1].

Recently, R. Gardner and V. Yaskin, together with the second and the third named authors constructed two bodies of revolution $K_{1}, K_{2}$ such that $K_{1}$ is origin-symmetric, $K_{2}$ is not origin-symmetric, but $M_{K_{1}} \equiv M_{K_{2}}$, thus answering the first version of Klee's question but not the second one (see [GRYZ]).

The main results we will present in this paper are the following.

Theorem 1. If $d=4$, there exists a convex body of revolution $K \subset \mathbb{R}^{d}$ satisfying $M_{K} \equiv$ const that is not a Euclidean ball.

Theorem 2. If $d \geq 4$ is even, there exist two essentially different convex bodies of revolution $K_{1}, K_{2} \subset \mathbb{R}^{d}$ such that $A_{K_{1}} \equiv A_{K_{2}}, M_{K_{1}} \equiv M_{K_{2}}$, and $P_{K_{1}} \equiv P_{K_{2}}$.

Theorem 1 answers the question of Klee in $\mathbb{R}^{4}$, and Theorem 2 answers the analogue of the question of Bonnesen in even dimensions.

Remark 1. Theorem 1 is actually true in all dimensions, but the construction for $d \neq 4$ is long and rather technical, so we will present it in a separate paper.

We borrowed the general idea of the construction of the bodies $K_{1}$ and $K_{2}$ in Theorem 2 from [RY], which attributes it to [GV] and [GSW]. It can be easily understood from the following illustration.
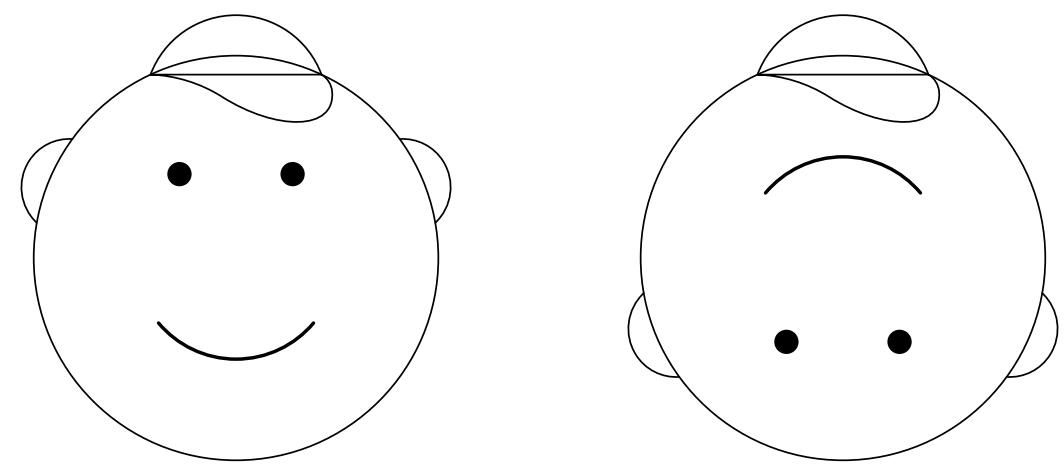

Figure 1. Two small-eared round faces in a cap

Here the "ears" and the "cap" will be made very small in order not to destroy the convexity of the bodies.

The paper is organized as follows. In Section 2 we reduce the problem to finding a non-trivial solution to two integral equations. In Section 3 we prove Theorem 1. In Section 4 we prove Theorem 2 . 


\section{Reduction to A SyStem of Integral EQUATions}

From now on, we assume that $d \geq 3$. We will be dealing with the bodies of revolution

$$
K_{f}=\left\{x \in \mathbb{R}^{d}: x_{2}^{2}+x_{3}^{2}+\ldots+x_{d}^{2} \leq f^{2}\left(x_{1}\right)\right\},
$$

obtained by the rotation of a smooth concave function $f$ supported on $[-1,1]$ about the $x_{1}$-axis.

Note that $K$ is rotation invariant, thus every its hyperplane section is equivalent to a section by a hyperplane with normal vector in the second quadrant of the $\left(x_{1}, x_{2}\right)$ plane.

Lemma 1. Let $L(\xi)=L(s, h, \xi)=s \xi+h$ be a linear function with slope $s$, and let $H(L)=\left\{x \in \mathbb{R}^{d}: x_{2}=L\left(x_{1}\right)\right\}$ be the corresponding hyperplane. Then the section $K \cap H(L)$ is of maximal volume iff

$$
\int_{-x}^{y}\left(f^{2}-L^{2}\right)^{(d-4) / 2} L=0,
$$

where $-x$ and $y$ are the first coordinates of the points at which $L$ intersects the graphs of $-f$ and $f$ respectively (see Figure 2).

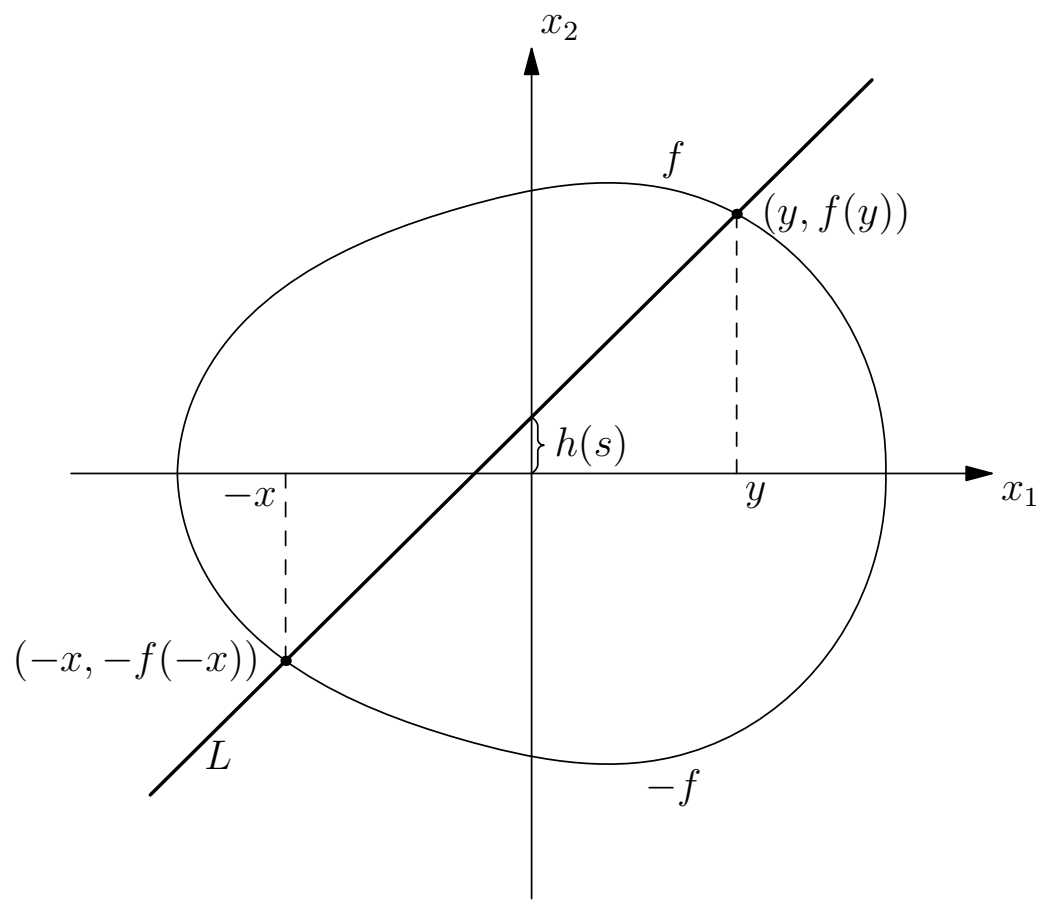

Figure 2. View of $K$ and $H(L)$ in $\left(x_{1}, x_{2}\right)$-plane.

Proof. Fix $s>0$. Observe that the slice $K \cap H(L) \cap H_{\xi}$ of $K \cap H(L)$ by the hyperplane $H_{\xi}=\left\{x \in \mathbb{R}^{d}: x_{1}=\xi\right\},-1<\xi<1$, is the $(d-2)$-dimensional Euclidean ball 
$\left\{\left(x_{3}, x_{4}, \ldots, x_{d}\right): x_{3}^{2}+\ldots+x_{d}^{2} \leq r^{2}\right\}$ of radius $r=\sqrt{f^{2}(\xi)-L^{2}(\xi)}$. Hence,

$$
\operatorname{vol}_{d-1}(K \cap H(L))=v_{d-2} \sqrt{1+s^{2}} \int_{-x(s)}^{y(s)}\left(f^{2}(\xi)-L^{2}(\xi)\right)^{(d-2) / 2} d \xi,
$$

where $v_{d-2}$ is the volume of the unit ball in $\mathbb{R}^{d-2}$.

The section $K \cap H(L)$ is of maximal volume iff

$$
\frac{d}{d h} \operatorname{vol}_{d-1}(K \cap H(L))=0
$$

where in the only if part we use the Theorem of Brunn, Ga. Computing the derivative, we conclude that for a given $s \in \mathbb{R}$, the section $K \cap H(L)$ is of maximal volume iff (4) holds.

Lemma 2. Let $L(s, \xi)=s \xi+h(s)$ be a family of linear functions parameterized by the slope s. For each $L$ in our family, define the hyperplane $H(L)$ by $H(L)=\{x \in$ $\left.\mathbb{R}^{d}: x_{2}=L\left(x_{1}\right)\right\}$, (see Figure 2). The corresponding family of sections is of constant d-1-dimensional volume iff

$$
\int_{-x}^{y}\left(f^{2}-L^{2}\right)^{(d-2) / 2}=\frac{\text { const }}{\sqrt{1+s^{2}}}, \quad \text { for all } \quad s>0 .
$$

Proof. The right hand side in (5) is constant iff (6) holds.

3. THE CASE $d=4$

Observe that when $d=4$, the system of equations (4), (6) simplifies to

$$
\int_{-x}^{y} L=0, \quad \text { and } \quad \int_{-x}^{y}\left(f^{2}-L^{2}\right)=\frac{\text { const }}{\sqrt{1+s^{2}}}, \quad \text { for all } \quad s>0 .
$$

In this case we will show that the maximal sections correspond to level intervals, see Proposition 1 below. We will also prove that the values of the maximal section function $M_{K}$ depend on the distribution function $t \rightarrow|\{f>t\}|$ only. More precisely, we have

Theorem 3. Let $d=4, K=\left\{x \in \mathbb{R}^{4}: x_{2}^{2}+x_{3}^{2}+x_{4}^{2} \leq f^{2}\left(x_{1}\right)\right\}$, and let

$$
u=u(s)=\left(-\frac{s}{\sqrt{1+s^{2}}}, \frac{1}{\sqrt{1+s^{2}}}, 0,0\right) \in \mathbb{S}^{3}, \quad s>0 .
$$

Then,

$$
M_{K_{f}}(u)=\pi \sqrt{1+s^{2}}\left(\frac{2}{3} t^{2}|\{f>t\}|+\int_{t}^{\infty} 2 \tau|\{f>\tau\}| d \tau\right),
$$

where $t$ is the unique solution of the equation $s=2 t /|\{f>t\}|$.

In particular, if $f_{1}$ and $f_{2}$ are equimeasurable (i.e., for every $\tau>0$, we have $\left.\left|\left\{f_{1}>\tau\right\}\right|=\left|\left\{f_{2}>\tau\right\}\right|\right)$, then $M_{K_{f_{1}}} \equiv M_{K_{f_{2}}}$. 
Theorem 3 is a simple consequence of the following two propositions.

Proposition 1. Let $f, s, u(s)$ be as in Theorem 3. Then the section of maximal volume in the direction $u(s)$ is the one that corresponds to the line joining $(-x,-t)$ and $(y, t)$, where $t$ is such that $s=2 t /|\{f>t\}|, 0<t<\max _{\xi \in[-1,1]} f(\xi)$, (see Figure 3).

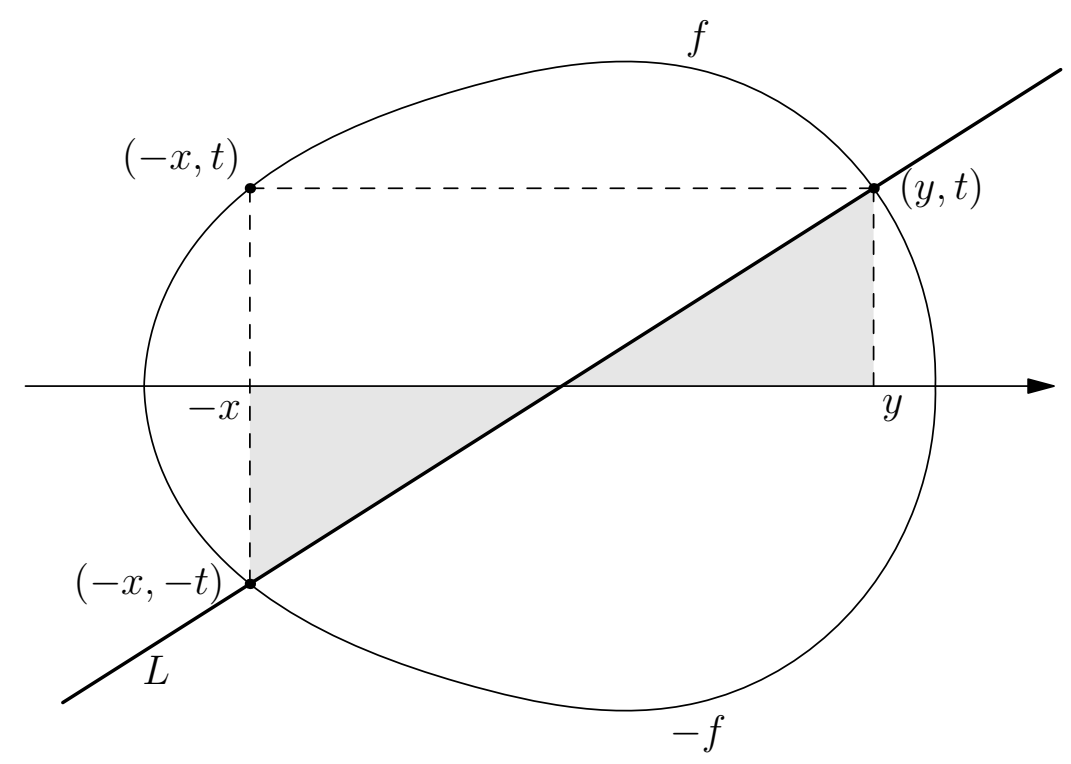

Figure 3. Maximal slice in $\mathbb{R}^{4}$

Proof. Fix $s>0$. Since the distribution function is decreasing to 0, there exists a unique $t$ satisfying $s=2 t /|\{f>t\}|$. To prove that

$$
\int_{-x}^{y} L(\xi) d \xi=0
$$

observe that two shaded triangles on Figure 3 are congruent.

Proposition 2. Let $K, f, t, x, y$ be as in the previous proposition, and let the line $L$ be passing through the points $(-x,-t),(y, t)$. Then (7) holds.

Proof. Note that

$$
\int_{-x}^{y} L^{2}=(x+y) \frac{t^{2}}{3}=\frac{t^{2}}{3}|\{f>t\}|
$$

and

$$
\int_{-x}^{y} f^{2}=\int_{\{f>t\}} f^{2}=t^{2}|\{f>t\}|+\int_{t}^{\infty} 2 \tau|\{f>\tau\}| d \tau .
$$


Proof of Theorem 1. Let $f_{o}(\xi)=\sqrt{1-\xi^{2}}, \xi \in[-1,1]$. Take a concave function $f$ on $[-1,1]$ such that $f \neq f_{o}$ and $f$ is equimeasurable with $f_{o}$.

\section{Proof of Theorem 2}

Let $\varphi$ and $\psi$ be two smooth functions supported on the intervals $D=\left[\frac{1}{2}-\delta, \frac{1}{2}+\delta\right]$ and $E=[1-\delta, 1]$ respectively, where $0<\delta<\frac{1}{8}$. Define

$$
f_{+}(\xi)=f_{o}(\xi)+\varepsilon \varphi(\xi)-\varepsilon \varphi(-\xi)+\varepsilon \psi(\xi),
$$

and

$$
f_{-}(\xi)=f_{o}(\xi)-\varepsilon \varphi(\xi)+\varepsilon \varphi(-\xi)+\varepsilon \psi(\xi),
$$

where $\varepsilon>0$ is so small that $f_{ \pm}$are concave on $[-1,1]$ (see Figure 4).

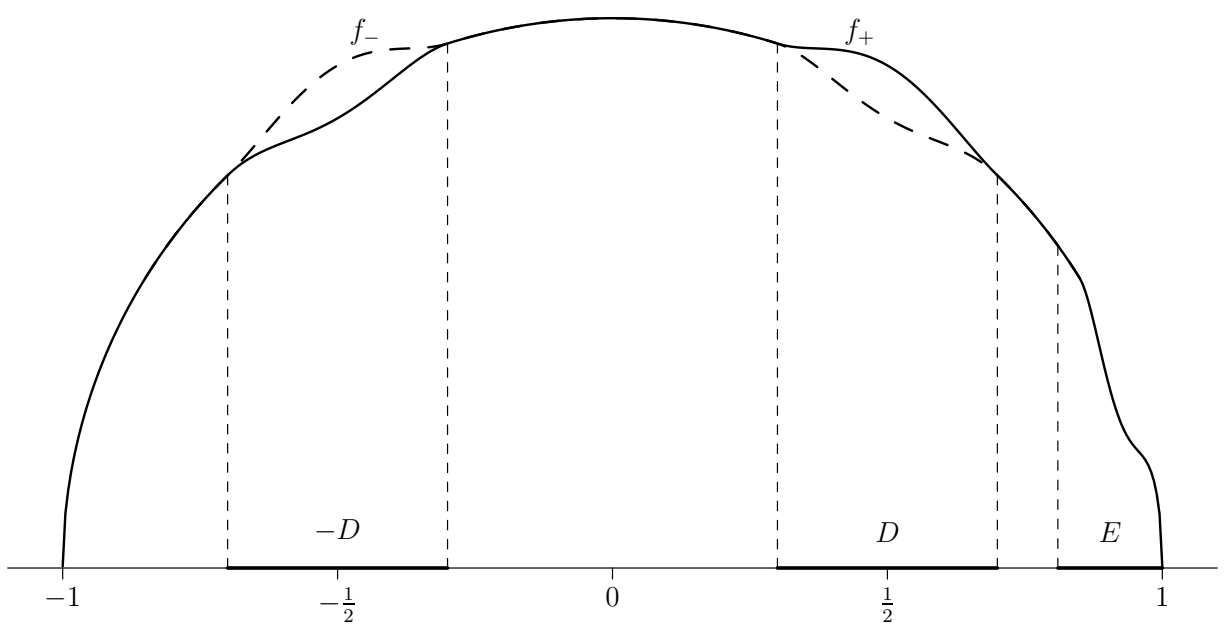

FiguRE 4. Graph of functions $f_{ \pm}$

Define $K_{1}=K_{f_{+}}$and $K_{2}=K_{f_{-}}$.

Observe that

(8) $f_{+}(\xi)=f_{-}(\xi) \forall \xi \in[-1,1] \backslash(D \cup(-D))$, and $f_{+}(\xi)=f_{-}(-\xi) \forall \xi \in D \cup(-D)$.

We can choose $\varepsilon$ so small that $K_{1}$ and $K_{2}$ are very close to the Euclidean ball, and the sections of the maximal volume of $K_{1}$ and $K_{2}$ are very close to the central sections of the ball.

In particular, the intersection points $x=x(s)$ and $y=y(s)$ satisfy

$$
x(s), y(s)>\frac{5}{8} \text { if } s \leq \frac{\sqrt{7}}{3}, \quad \text { and } \quad x(s), y(s)<\frac{7}{8} \text { if } s \geq \frac{\sqrt{7}}{3},
$$

for both bodies.

First, we show that $P_{K_{1}} \equiv P_{K_{2}}$ and $A_{K_{1}} \equiv A_{K_{2}}$.

Observe that we have $h_{K_{1}}(u)=h_{K_{2}}(u)$ and $\rho_{K_{1}}(u)=\rho_{K_{2}}(u)$ for all directions $u=\left(\xi, \sqrt{1-\xi^{2}}, 0,0, \ldots, 0\right) \in \mathbb{S}^{d-1}, \xi \in[0,1] \backslash D$. Observe also that $h_{K_{1}}(u)=h_{K_{2}}(-u)$ 
and $\rho_{K_{1}}(u)=\rho_{K_{2}}(-u)$ for all directions $u=\left(\xi, \sqrt{1-\xi^{2}}, 0,0, \ldots, 0\right) \in \mathbb{S}^{d-1}, \xi \in D$. Hence, the non-ordered pairs

$$
\left\{h_{K_{1}}(u), h_{K_{1}}(-u)\right\}, \quad \text { and } \quad\left\{h_{K_{2}}(u), h_{K_{2}}(-u)\right\}
$$

coincide for all $u \in \mathbb{S}^{d-1}$, and so do the pairs

$$
\left\{\rho_{K_{1}}(u), \rho_{K_{1}}(-u)\right\}, \quad \text { and } \quad\left\{\rho_{K_{2}}(u), \rho_{K_{2}}(-u)\right\} .
$$

By the result of Goodey, Schneider and Weil, [GSW], we have $P_{K_{1}} \equiv P_{K_{2}}$. Also,

$$
\begin{gathered}
A_{K_{1}}(\theta)=\frac{1}{d-1} \int_{\mathbb{S}^{d-1} \cap \theta^{\perp}} \frac{\rho_{K_{1}}^{d-1}(-u)+\rho_{K_{1}}^{d-1}(u)}{2} d \sigma(u)= \\
\frac{1}{d-1} \int_{\mathbb{S}^{d-1} \cap \theta^{\perp}} \frac{\rho_{K_{2}}^{d-1}(-u)+\rho_{K_{2}}^{d-1}(u)}{2} d \sigma(u)=A_{K_{2}}(\theta)
\end{gathered}
$$

for all $\theta \in \mathbb{S}^{d-1}$.

It remains to show that

$$
M_{K_{1}} \equiv M_{K_{2}} .
$$

Assume first that $d=4$. The functions $f_{+}$and $f_{-}$are equimeasurable, so 10 follows from Theorem 3 .

Let now $d \geq 6$ be even. Note that in this case, $p=\frac{d-2}{2} \in \mathbb{N}$.

We claim that we can choose $\varphi$ such that (10) holds. This result will be a consequence of the following two propositions.

Proposition 3. If $\varepsilon$ is small enough, then for

we have

$$
u=u(s)=\left(-\frac{s}{\sqrt{1+s^{2}}}, \frac{1}{\sqrt{1+s^{2}}}, 0,0, \ldots, 0\right) \in \mathbb{S}^{d-1}
$$

$$
M_{K_{1}}(u)=M_{K_{2}}(u)
$$

provided $s \geq \frac{\sqrt{7}}{3}$.

Proof. By (9), if the sections $K_{1} \cap H\left(L_{1}\right), K_{2} \cap H\left(L_{2}\right)$ are the sections of the maximal volume, corresponding to the same slope $s \geq \frac{\sqrt{7}}{3}$, then, they are the sections of two symmetric (to each other) bodies corresponding to $\psi=0$. Hence, (11) holds by symmetry.

To formulate the second proposition we will need the following result, which is a consequence of the Borsuk-Ulam Theorem.

Lemma 3. There exists $\varphi \neq 0$ such that

$$
\int_{-\frac{5}{8}}^{\frac{5}{8}} f_{+}^{2 j}(\xi) \xi^{l} d \xi=\int_{-\frac{5}{8}}^{\frac{5}{8}} f_{-}^{2 j}(\xi) \xi^{l} d \xi
$$

for all $j=0, \ldots, p$ and $l=0, \ldots, 2(p-j)$. 
Proof. We will choose $\varphi$ using the Borsuk-Ulam Theorem.

For $j$ and $l$ as above, consider the vector $\mathbf{a}=\mathbf{a}(\varphi)$ with coordinates

$$
a_{j, l}(\varphi)=\int_{-\frac{5}{8}}^{\frac{5}{8}} f_{+}^{2 j}(\xi) \xi^{l} d \xi-\int_{-\frac{5}{8}}^{\frac{5}{8}} f_{-}^{2 j}(\xi) \xi^{l} d \xi .
$$

We will view this vector as an element of $\mathbb{R}^{n(p)}$ with appropriately chosen $n(p)$.

For $x=\left(x_{0}, x_{1}, \ldots, x_{n(p)}\right) \in \mathbb{S}^{n(p)}$ define $\varphi_{x}=\sum_{j=0}^{n(p)} x_{j} \varphi_{j}$, where $\varphi_{j}$ are smooth not identically zero functions with pairwise disjoint supports contained in $D$, and let

$$
\text { B : }\left(x_{0}, x_{1}, \ldots, x_{n(p)}\right) \rightarrow \varphi_{x} \rightarrow \mathbf{a}\left(\varphi_{x}\right)
$$

be our map from $\mathbb{S}^{n(p)}$ to $\mathbb{R}^{n(p)}$. By the definition of $f_{ \pm}$the map B is odd. Hence, by the Borsuk-Ulam Theorem, one can choose $x$ (and hence $\varphi_{x}$ ) in such a way that $\mathbf{a}\left(\varphi_{x}\right)=\mathbf{0}$.

Proposition 4. Let $L=L(s, h, \xi)$ be as above and let $0 \leq s \leq \frac{\sqrt{7}}{3}$. Then,

$$
\int_{-x}^{y}\left(f_{-}^{2}-L^{2}\right)^{p}=\int_{-x}^{y}\left(f_{+}^{2}-L^{2}\right)^{p}
$$

Moreover,

$$
\int_{-x}^{y}\left(f_{+}^{2}-L^{2}\right)^{p-1} L=0 \quad \text { if and only if } \quad \int_{-x}^{y}\left(f_{-}^{2}-L^{2}\right)^{p-1} L=0 .
$$

In particular, 11) holds for $0 \leq s \leq \frac{\sqrt{7}}{3}$.

Proof. We start with the proof of (13). We open parentheses and observe that all we need to prove is

$$
\int_{-x(s)}^{y(s)} f_{+}^{2 j}(\xi) \xi^{l} d \xi=\int_{-x(s)}^{y(s)} f_{-}^{2 j}(\xi) \xi^{l} d \xi
$$

for all $0 \leq s \leq \frac{\sqrt{7}}{3}$, and for all $j=0, \ldots, p$ and $l=0, \ldots, 2(p-j)$. By $(9)$, this follows from $(12)$ since $f_{+}(\xi)=f_{-}(\xi)$ for $|\xi| \geq \frac{5}{8}$.

Similarly, (12) implies (14) for $j=0, \ldots, p-1$ and $l=0, \ldots, 2(p-1-j)+1$.

Thus, (10) follows from Propositions 3, 4. This finishes the proof of the Theorem.

\section{REFERENCES}

[BF] T. Bonnesen And Fenchel, Theory of convex bodies, Moscow, Idaho, 1982.

[Ga] R.J. Gardner, Geometric tomography, Second edition. Encyclopedia of Mathematics and its Applications, 58, Cambridge University Press, Cambridge, 2006.

[GV] R.J. Gardner, A. VolČIČ, Tomography of convex and star bodies, Adv. Math. 108 (1994), no. $2,367-399$. 
[GRYZ] R.J. Gardner, D. Ryabogin, V. Yaskin, and A Zvavitch, On a problem of Klee, to appear in J. Diff. Geometry.

[GSW] P. Goodey, R. Schneider and W. Weil, On the determination of convex bodies by projection functions, Bull. London Math. Soc.29 (1997), 82-88.

[Kl1] V. KLEE, Is a body spherical if its HA-measurements are constant?, Amer. Math. Monthly 76 (1969), 539-542.

[RY] D. RYabogin AND V. YASkin, On counterexamples in questions of unique determination of convex bodies, to appear in Proc. of AMS.

Department of Mathematics, Kent State University, Kent, OH 44242, USA

E-mail address: nazarov@math.kent.edu

E-mail address: ryabogin@math.kent.edu

E-mail address: zvavitch@math.kent.edu 